\title{
Histone deacetylase 3, not histone deacetylase 2, interacts with the major immediate early locus of human cytomegalovirus
}

\author{
Ying Huang ${ }^{1 \dagger}$, Qiyi Tang ${ }^{2^{*}}$, Michael Nguyen ${ }^{1}$, Kalpana Dulal ${ }^{1}$, Weijia Wang ${ }^{1}$ and Hua Zhu ${ }^{1 *}$
}

\begin{abstract}
Evidence suggests that genome chromatinization and the posttranslational modification of histones are involved in the regulation of viral gene expression, including the human cytomegalovirus (HCMV). We performed a ChIP-onChip assay to determine whether histone deacetylases (HDACs) interact with HCMV genomic DNA on a global level. Surprisingly, we found that HDAC3, but not HDAC2, interacts not only with the major immediate early (MIE) promoter but also with the entire MIE locus, suggesting a heterogeneous interaction of HDAC3 with HCMV DNA. The interaction of HDAC3 with the MIE region is related to inhibition of viral replication because HDAC3 inhibitors enhanced HCMV replication.
\end{abstract}

Human cytomegalovirus (HCMV) is a ubiquitous virus and infects a majority of the general population (50-90\%) [1]. The fact that the incidence of cytomegalic inclusion disease (CID) is intimately related to viral burden suggests that the inhibition of viral production by the specific repression of viral gene expression will reduce the occurrence of CID [2]. Understanding the mechanism of HCMV gene regulation is the pre-requisite for developing drugs that interfere with viral replication by repressing viral gene expression. The HCMV major immediate early (MIE) gene products, IE1 and IE2, are among the first de novo-expressed viral proteins that are required for subsequent viral gene expression and hence viral replication [3]. IE1 and IE2 mRNAs are encoded by the major IE locus that spans from 169 to $175 \mathrm{kbp}$ of the viral genome and are produced by alternative splicing and differential polyadenylation [4-6]. IE1 and IE2 share the first 85 amino acids [1]. MIE genes are controlled by a strong promoter/enhancer that contains many regulatory elements $[3,7]$.

\footnotetext{
* Correspondence: qtang@psm.edu; zhuhu@umdnj.edu

† Contributed equally

'Department of Microbiology and Molecular Genetics, UMDNJ-New Jersey Medical School, 225 Warren Street, Newark, NJ, 07101, USA

${ }^{2}$ Department of microbiology/AIDS program, Ponce School of Medicine, 395

Zona Industrial Reparadara-2, Ponce, PR, 00716-2348, USA

Full list of author information is available at the end of the article
}

Different cellular mechanisms have been found to play roles in inhibiting viral gene expression, and one of the most prominent ones is gene silencing through viral DNA chromatinization (also called chromatin remodeling) [8], a procedure carried out by histone or histonerelated proteins, such as histone acetylase (HAT) and histone deacetylase (HDAC). Several posttranslational modifications of histone proteins have been defined to be involved in chromatin remodeling, including acetylation by HAT, deacetylation by HDAC, SUMOylation by SUMO (Small Ubiquitin-like Modifier)-related pathway, deSUMOylation by SENP (a SUMO-specific protease) family enzymes, phosphorylation by kinase pathways, and methylation via methylases [9-13]. Those enzymatic pathways orchestrate to regulate cellular gene transcription and are termed as epigenetic codes $[10,13]$. Viral gene transcription requires cellular machinery, which is probably also regulated by cellular gene regulatory pathways. Histones are abundant nuclear proteins and have been shown to bind with HCMV genomic DNA [14]. Therefore, it was reasonable to propose that chromatin remodeling of viral DNA takes place in the nucleus, which speculation was validated when the fact that HDAC inhibitors can promote cytomegalovirus production was also confirmed [15-17].

How HCMV strips off the cellular proteins in order for the virus to replicate its own DNA is not fully understood. Recent studies have shown that HCMV and murine CMV 
(MCMV) major immediate early proteins, IE1 and IE2 (or IE3 for MCMV), interact with HDAC1, 2, and 3, and HDAC inhibitors enhance viral production [17-21], and dynamic chromatin modification of the MIE promoter and other viral promoters has been shown. However, interaction of HDACs with the viral genome has not been clearly demonstrated [22]. In this study, we performed chromatin immunoprecipitation followed by microarray on an HCMV DNA chip (ChIP-on-chip) assay to demonstrate the interaction of HCMV DNA with HDACs. To our surprise, we found that HDAC3, but not HDAC2, interacts specifically with the MIE locus, which suggests a heterogeneous interaction of HDAC3 with HCMV genomic DNA. In addition, we found that the interactions of HDAC3 with the MIE locus might relate to the modulation of viral replication because HDAC3 inhibitors can significantly enhance viral growth.

The chromatinization of viral DNA after its having entered the nucleus has been noted not only in latently infected viruses such as EBV and KSHV (the genomes of which are tethered to cellular chromosomes) but also in the lytic infection of HCMV $[8,23,24]$. On the other hand, histone proteins have not been found in herpesvirus virons [25]. Therefore, the chromatinization of HCMV DNA must be temporary and dynamic. We wonder 1) whether the HDACs are bound to the HCMV DNA, and 2) if so, where they interact and whether the interaction is homogenous or heterogeneous. In order to answer these questions, we performed a ChIP-on-chip assay.

The human foreskin fibroblast cells (HFF) were infected with HCMV at an MOI of 5 . The cells were fixed at 24 hours postinfection with $1 \%$ paraformaldehyde. The chromatin immunoprecipitation (ChIP) inputs were prepared and performed using the commercial kit (EZ ChIP, Upstate Cell Signal Solutions), according to the manufacturer's protocol. The antibodies used for ChIP assays include anti-HDAC2 (clone 3F3), antiHDAC3 (clone 3G6, Upstate USA, Inc.), and normal IgG (as a negative control).

To generate an HCMV genomic microarray for the ChIP-on-chip assay, an entire HCMV (Toledo strain) genomic DNA was subdivided into 593 small DNA fragments and amplified by PCR (primers are listed in Additional file 1, Table S1). Each PCR fragment was 500 bp long with $100 \mathrm{bp}$ overlapping the adjacent fragments. The PCR products were verified by agarose gels, purified, quantified, and printed on glass slides, as described [26]. Each DNA fragment was spotted in triplicate on each array. The printing quality of the array was controlled by hybridizing the array with a Cyanine 3-dUTPlabeled random 9-mer, and the slides were scanned using an Axon 4000A scanner at $532 \mathrm{~nm}$. Figure 1 shows that all 593 HCMV genomic fragments were printed on the microarray relatively evenly.

Since chromatin immunoprecipitation yields a small amount of DNA, PCR amplifications are required before labeling steps. First, precipitated input DNA was randomly primed with a sequence-tagged oligonucleotide (GTTTCCCAGTCACGATCNNNN NNNNN) to generate templates with a specific tag at both ends for subsequent PCR. Then, a specific primer (GTTTCCCAG-TCACGATC) was used to amplify the templates previously generated. The final amplification consisted of additional PCR cycles to incorporate aminoallyl-dUTP. The amplified products were then labeled with Alexa Flour 647 or Alexa Flour 555 and applied to a DNA array of HCMV. The hybridization was performed according to manufacture's instruction (Corning UltraGAPS ${ }^{\mathrm{TM}}$ ).

The slides were scanned using an Axon 4000A scanner. Images were analyzed using GenePix Pro 5.1. A normalization factor was determined using the ratio of the median signal intensity of the cy5 and cy3 channels, and all data sets were normalized to a single medium intensity. A $\log 2$ signal intensity ratio for each fragment was calculated for the ChIP samples that were pulled down by monoclonal antibody against HDAC3 (or HDAC2 or histone H3) to that by normal IgG. To correct for the biases in the ChIP procedure, all log ratios were normalized by subtracting the average log ratio across the whole array. We considered that a spot was enriched if the signal intensity from ChIP sample of anti-HDAC3 was 4-fold greater than the corrected signal from the IgG ChIP sample.

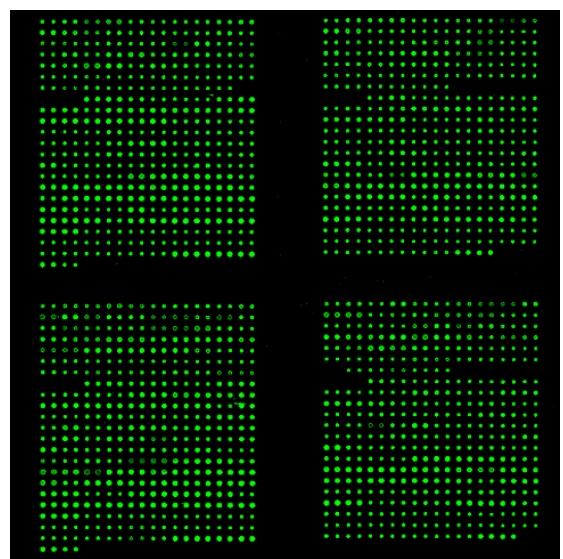

Figure $1 \mathrm{HCMV}$ array printing quality control. The printing quality was checked by hybridizing the array with a Cyanine 3-dUTP-labeled random 9-mer, and the slides were scanned using an Axon 4000A scanner at $532 \mathrm{~nm}$. The image showed that all 593 HCMV genomic fragments were printed on the microarray relatively evenly. 
To our surprise, we found that microarray spots corresponding to the DNA within the MIE locus interacted with HDAC3 only, not HDAC2 (Figure 2A, black arrows and data not shown). The MIE locus is only specific HDAC3-interacting region found in this study. Some non-specific hybridization signals were detected, as is indicated by the grey arrows.

To further confirm the ChIP-on-chip results, we performed a series PCR reactions using primers amplifying HCMV genomic fragments 113 to 140 (Figure 2,
Additional file 1, Table S1). As shown in Figure 2B, all HCMV genomic fragments were present in the ChIP input sample; IgG and HDAC2 did not specifically interact with the HCMV genome; histone $\mathrm{H} 3$ interacted with the entire viral DNA tested; and most importantly, HDAC3 interacted with fragment 47-53 kb of the Toledo genome corresponding to the HCMV MIE locus (Figure $2 \mathrm{C}$, green). To confirm that the anti-HDAC2 antibody is effective for the ChIP assay, we performed another ChIP assay using anti-HDAC2, - HDAC3

A

HDAC3: Green; IgG: Red

HDAC3: Red; IgG: Green
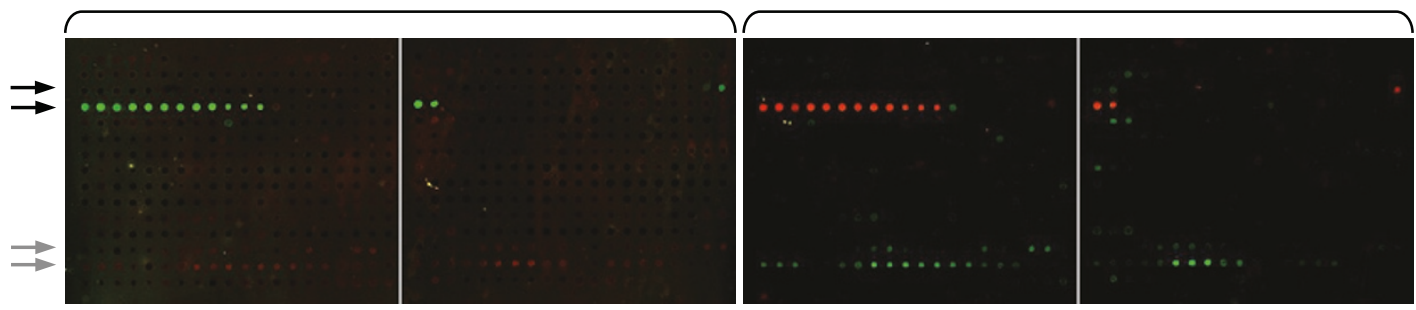

B

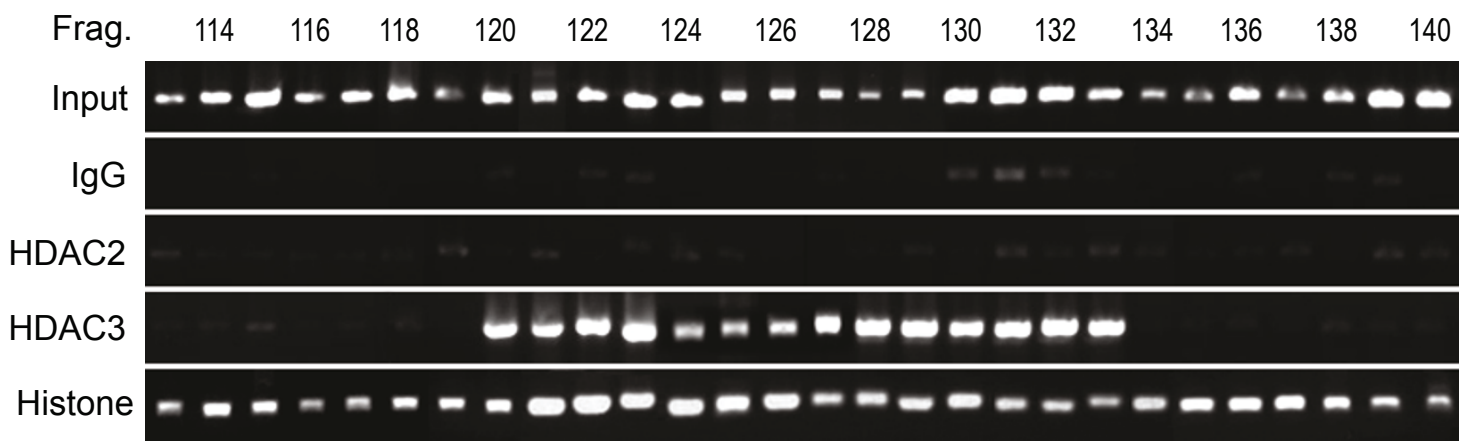

C

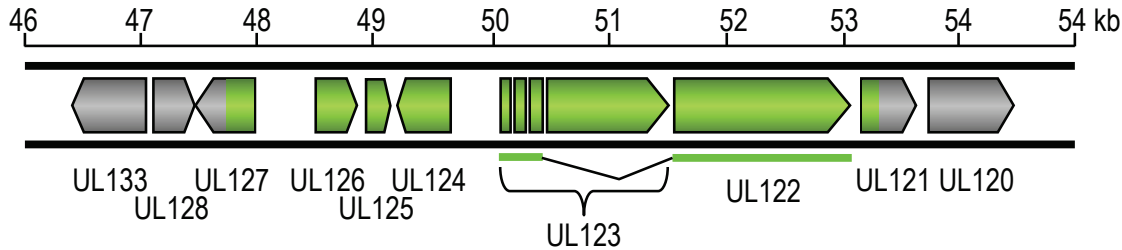

D

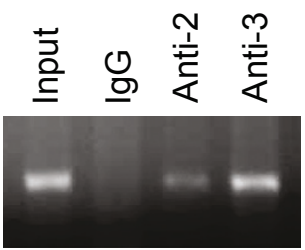

Figure 2 HDAC3 but not HDAC2 interacts with the MIE locus of HCMV. A. ChIP on Chip assay. HFF cells were infected with the Toledo strain at MOI 5, and the DNA samples from the infected cells were prepared by using the EZ-ChIP commercial kit. Anti-HDAC2, anti-HDAC3, and normal IgG (as a negative control) antibodies were used to precipitate the fragmented Protein-DNA complexes. The DNA fragments pulled down with HDAC3 and IgG antibody were amplified and labeled with Alexa Flour 555 and Alexa Flour 647, respectively and vice-versa. The labeled DNA was applied to the HCMV genomic Microarray containing the entire HCMV (Toledo strain) genomic DNA divided into 593 small fragments. The array was scanned at $532 \mathrm{~nm}$ (for Cy3) and $635 \mathrm{~nm}$ (for Cy5). HDAC3 was detected only in the MIE locus as indicated with black arrows. The grey arrows indicate no-specific hybridizations. B. PCR verification of the ChIP-on-chip assay. A series of PCR reactions covering the MIE region was performed on the sample before ChIP (Input) and ChIP samples, as indicated on left. C. HDAC3-interacting map. The ORFs spanning the MIE locus are shown. HDAC3-interacting region is indicated as green. D. HDAC2/3 ChIP on hTERT promoter. Mrc-5 cells were crosslinked and ChIP samples were made using the EZ-ChIP commercial kit. Anti-HDAC2 (Anti-2), anti-HDAC3 (Anti-3), and normal IgG (as a negative control) antibodies were used to precipitate the fragmented Protein-DNA complexes. PCR was performed to detect the precipitated hTERT promoter DNA using the primers and PCR protocol as reported [27]. 
antibodies to bind ChIP samples made from uninfected Mrc-5 cells. The eluted DNA samples were applied for running PCR against hTERT promoter as reported by Straat et al. As shown in Figure 2D, HDAC2 bound to hTERT promoter, which is consistent with the report [27].

Since HDAC3, like HDAC1 and 2, is one of the major components of nuclear co-repressors (DNA-remodeling complexes) inhibiting gene expression, HDAC inhibitors should be able to counter HDAC3's repressive effects on gene expression so that they can promote HCMV viral replication. To confirm our speculations, we infected HFF cells with HCMV (at MOIs of 0.1 and 5) in either the presence or absence of the HDAC inhibitor, Trichostatin A (TSA). Growth-curve analysis showed that the HDAC inhibitor significantly accelerated viral replication (Figure 3).

We demonstrated that HDAC3 but not HDAC2 interacts with the HCMV MIE locus. The reason that HDAC2 was not detected in our assay could be due to the fact that HDAC2 is known to interact with IE2, and this binding suppresses the repressive effect of HDAC2 [20]. At $24 \mathrm{hrs}$ postinfection, the genomic region around the MIE may need to stay repressed,
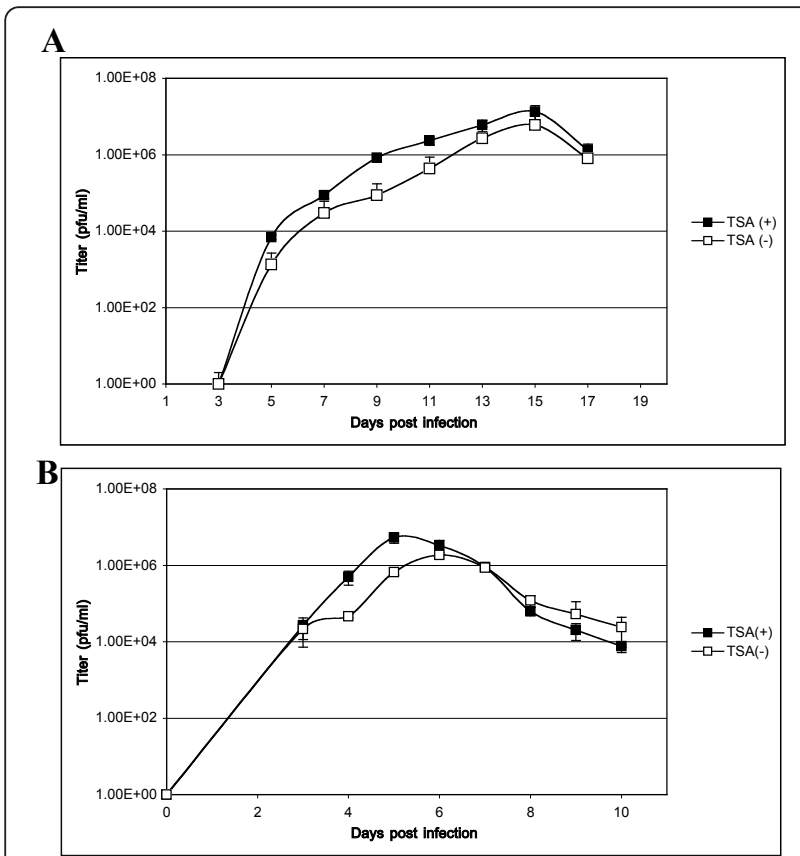

Figure 3 HDAC inhibitors enhance viral replication. HFF cells were infected with the AD169 strain of HCMV at MOI $=0.1$ (panel A) and $\mathrm{MOI}=5$ (panel B) both in the presence and absence of TSA $(250 \mathrm{nM})$. At different times postinfection, virus titer was determined using the plaque formation assay. Data are expressed as the average of three independent experiments. As seen in panel $A$ and $B$, the production of infectious viral particles with TSA is approximately 10 -fold higher at its maximum than without TSA. which could be accomplished by the action of HDAC3. This binding of HDAC3 to not only the MIE locus but also the genes upstream of MIE is intriguing. The region between the MIE and UL127 has been named as a unique region without any known function. This region is known to have binding sites for various cellular repressor proteins that help to repress the transcription of UL127. It is known that the promoter of UL127 gene is silenced during productive infection in fibroblasts [28]. The UL126 gene has been reported as a latency-associated gene [29]. UL124 is a putative membrane glycoprotein that may be expressed late in the infection process [30]. It will be interesting to see how the HDACs bind with those genomic regions at later times of infection.

HDAC3 has been shown to be a repressor of the viral MIE promoter [14]. A significant increase in viral replication in the presence of the HDAC inhibitor demonstrates that the binding of HDAC3 causes inhibition of viral replication. This inhibition seems to be more pronounced at a low MOI. Host cells contain several proteins including gene expression suppressors that form a defensive arm against viral infection [31]. HCMV has evolved strategies against cellular defense. We speculate that HCMV infection must reduce HDAC activity. Both IE1 and IE2 of HCMV were reported to functionally interact with HDACs [14, 18-20], and IE1 of MCMV is particularly adept at reducing HDAC activity [17]. These observations and our finding of an increase in viral replication in the presence of TSA further confirm the IE2-mediated repression of the MIE promoter by the recruitment of chromatin remodeling factors.

\section{Additional material}

Additional file 1: Table S1. PCR primer sequences for HCMV genomic microarray construction.

\begin{abstract}
Abbreviations
HCMV: (human cytomegalovirus); HDACs: (histone deacetylases); MIE: (major immediate early); CID: (cytomegalic inclusion disease); SUMO: (small ubiquitin-like modifier); SENP: (SUMO-specific protease); HFF: (human foreskin fibroblast cells); TSA: (Trichostatin A).
\end{abstract}

\section{Acknowledgements}

This work was supported by NIH grant Al050709 (H.Z.), a Pilot grant from the Research Center for Minority Institutes (RCMI) Program (2G12RR00305024) (Q.T.), and American Cancer Society grant RSG-090289-01-MPC (Q.T). We are grateful to Bob Ritchie (RCMI, 2G12RR003050-24) for English editing.

\section{Author details}

${ }^{1}$ Department of Microbiology and Molecular Genetics, UMDNJ-New Jersey Medical School, 225 Warren Street, Newark, NJ, 07101, USA. ²Department of microbiology/AIDS program, Ponce School of Medicine, 395 Zona Industrial Reparadara-2, Ponce, PR, 00716-2348, USA. 


\section{Authors' contributions}

YH developed HCMV genomic microarray, carried out growth curve, PCR and data analyses QT carried out ChIP-on-Chip assay and drafted the manuscript. MN developed HCMV genomic microarray and carried out ChIPon-Chip assay. KD carried out growth curve analysis. WW participated initial studies and carried out growth curve analysis. $\mathrm{HZ}$ conceived of the study, and participated in its design, coordination and manuscript preparation. All authors read and approved the final manuscript.

\section{Competing interests}

The authors declare that they have no competing interests.

Received: 14 October 2010 Accepted: 31 March 2011

Published: 31 March 2011

\section{References}

1. Mocarski E: Cytomegaloviruses and Their Replication. In Fields Virology. Volume 2.. 5 edition. Edited by: Knipe DMaH. P.M. Philadelphia: Lippincott Williams 2007:2629-2673.

2. Fowler KB, Boppana SB: Congenital cytomegalovirus (CMV) infection and hearing deficit. J Clin Virol 2006, 35:226-231.

3. Stenberg RM: The human cytomegalovirus major immediate-early gene. Intervirology 1996, 39:343-349.

4. Davison AJ, Dolan A, Akter P, Addison C, Dargan DJ, Alcendor DJ, McGeoch DJ, Hayward GS: The human cytomegalovirus genome revisited: comparison with the chimpanzee cytomegalovirus genome. J Gen Virol 2003, 84:17-28.

5. Murphy E, Shenk T: Human cytomegalovirus genome. Curr Top Microbiol Immunol 2008, 325:1-19.

6. Murphy E, Rigoutsos I, Shibuya T, Shenk TE: Reevaluation of human cytomegalovirus coding potential. Proc Natl Acad Sci USA 2003, 100:13585-13590.

7. Stinski MF, Thomsen DR, Stenberg RM, Goldstein LC: Organization and expression of the immediate early genes of human cytomegalovirus. J Virol 1983, 46:1-14.

8. Nitzsche A, Paulus C, Nevels M: Temporal dynamics of cytomegalovirus chromatin assembly in productively infected human cells. J Virol 2008, 82:11167-11180

9. $\mathrm{Ng} \mathrm{HH}$, Bird A: Histone deacetylases: silencers for hire. Trends Biochem Sci 2000, 25:121-126.

10. Johnson ES: Protein modification by SUMO. Annu Rev Biochem 2004, 73:355-382.

11. Lyst MJ, Stancheva I: A role for SUMO modification in transcriptional repression and activation. Biochem Soc Trans 2007, 35:1389-1392.

12. Minucci S, Pelicci PG: Histone deacetylase inhibitors and the promise of epigenetic (and more) treatments for cancer. Nat Rev Cancer 2006, 6:38-51.

13. Sims RJ, Reinberg D: Is there a code embedded in proteins that is based on post-translational modifications? Nat Rev Mol Cell Biol 2008, 9:815-820.

14. Murphy JC, Fischle W, Verdin E, Sinclair JH: Control of cytomegalovirus Iytic gene expression by histone acetylation. EMBO J 2002, 21:1112-1120.

15. Michaelis M, Suhan $T$, Reinisch A, Reisenauer A, Fleckenstein C, Eikel D, Gumbel H, Doerr HW, Nau H, Cinatl J Jr: Increased replication of human cytomegalovirus in retinal pigment epithelial cells by valproic acid depends on histone deacetylase inhibition. Invest Ophthalmol Vis Sci 2005, 46:3451-3457.

16. Vanniasinkam T, Ertl H, Tang Q: Trichostatin-A enhances adaptive immune responses to DNA vaccination. J Clin Virol 2006, 36:292-297.

17. Tang Q, Maul GG: Mouse cytomegalovirus immediate-early protein 1 binds with host cell repressors to relieve suppressive effects on viral transcription and replication during lytic infection. J Virol 2003, 77:1357-1367.

18. Nevels M, Paulus C, Shenk T: Human cytomegalovirus immediate-early 1 protein facilitates viral replication by antagonizing histone deacetylation. Proc Natl Acad Sci USA 2004, 101:17234-17239.

19. Reeves M, Murphy J, Greaves R, Fairley J, Brehm A, Sinclair J: Autorepression of the human cytomegalovirus major immediate-early promoter/enhancer at late times of infection is mediated by the recruitment of chromatin remodeling enzymes by IE86. J Virol 2006, 80:9998-10009
20. Park JJ, Kim YE, Pham HT, Kim ET, Chung YH, Ahn JH: Functional interaction of the human cytomegalovirus IE2 protein with histone deacetylase 2 in infected human fibroblasts. J Gen Virol 2007. 88:3214-3223.

21. Martinez FP, Cosme RS, Tang Q: Murine Cytomegalovirus Major Immediate Early Protein 3 (IE3) Interacts with Cellular and Viral Proteins in the Viral DNA Replication Compartments and Is Important for Early Gene Activation. J Gen Virol 2010, 91(Pt 11):2664-76.

22. Cuevas-Bennett $\mathrm{C}$, Shenk T: Dynamic histone $\mathrm{H} 3$ acetylation and methylation at human cytomegalovirus promoters during replication in fibroblasts. J Virol 2008, 82:9525-9536.

23. Lu F, Zhou J, Wiedmer A, Madden K, Yuan Y, Lieberman PM: Chromatin remodeling of the Kaposi's sarcoma-associated herpesvirus ORF50 promoter correlates with reactivation from latency. J Virol 2003, 77:11425-11435

24. Zhou J, Chau CM, Deng Z, Shiekhattar R, Spindler MP, Schepers A Lieberman PM: Cell cycle regulation of chromatin at an origin of DNA replication. EMBO J 2005, 24:1406-1417.

25. Varnum SM, Streblow DN, Monroe ME, Smith P, Auberry KJ, Pasa-Tolic L, Wang D, Camp DG, Rodland K, Wiley S, et al: Identification of proteins in human cytomegalovirus (HCMV) particles: the HCMV proteome. J Virol 2004, 78:10960-10966.

26. Yang S, Ghanny S, Wang W, Galante A, Dunn W, Liu F, Soteropoulos P, Zhu $H$ : Using DNA microarray to study human cytomegalovirus gene expression. J Virol Methods 2006, 131:202-208.

27. Straat K, Liu C, Rahbar A, Zhu Q, Liu L, Wolmer-Solberg N, Lou F, Liu Z, Shen J, Jia J, et al: Activation of telomerase by human cytomegalovirus. J Natl Cancer Inst 2009, 101:488-497.

28. Lashmit $P E$, Lundquist CA, Meier JL, Stinski MF: Cellular repressor inhibits human cytomegalovirus transcription from the UL127 promoter. I Virol 2004, 78:5113-5123.

29. Kondo K, Kaneshima H, Mocarski ES: Human cytomegalovirus latent infection of granulocyte-macrophage progenitors. Proc Natl Acad Sci USA 1994, 91:11879-11883.

30. Landini MP, Lazzarotto T, Xu J, Geballe AP, Mocarski ES: Humoral immune response to proteins of human cytomegalovirus latency-associated transcripts. Biol Blood Marrow Transplant 2000, 6:100-108.

31. Kalejta RF: Functions of human cytomegalovirus tegument proteins prior to immediate early gene expression. Curr Top Microbiol Immunol 2008, 325:101-115.

doi:10.1186/1743-422X-8-151

Cite this article as: Huang et al:: Histone deacetylase 3, not histone deacetylase 2 , interacts with the major immediate early locus of human cytomegalovirus. Virology Journal 2011 8:151.

\section{Submit your next manuscript to BioMed Central and take full advantage of:}

- Convenient online submission

- Thorough peer review

- No space constraints or color figure charges

- Immediate publication on acceptance

- Inclusion in PubMed, CAS, Scopus and Google Scholar

- Research which is freely available for redistribution

Submit your manuscript at www.biomedcentral.com/submit
C Biomed Central 